Artículo científico

Volumen 33(2): Artículo 47078, 2022

e-ISSN 2215-3608, doi:10.15517/am.v33i2.47078

https://revistas.ucr.ac.cr/index.php/agromeso/index

\title{
Perfil mineral de los alimentos importados para mascotas en Costa Rica ${ }^{1}$
}

\section{Mineral profile of imported pet food in Costa Rica}

\author{
André Cedeño-López ${ }^{2}$, Rodolfo WingChing-Jones ${ }^{2}$
}

1 Recepción: 21 de mayo, 2021. Aceptación: 7 de septiembre, 2021. Este trabajo formó parte del proyecto de graduación del primer autor para obtener la Licenciatura en Ingeniería Agronómica con énfasis en Zootecnia de la Universidad de Costa Rica, financiada por la Universidad de Costa Rica, San José, Costa Rica.

2 Universidad de Costa Rica, Escuela de Zootecnia, San José, Costa Rica. andre.cedeno@ucr.ac.cr (https://orcid.org/0000-0003-3436-5940), rodolfo.wingching@ucr.ac.cr (autor para la correspondencia; https://orcid.org/0000-0002-8009-2210).

\section{Resumen}

Introducción. El contenido mineral de los alimentos para mascotas no siempre está presente en la etiqueta. Es importante conocer esta información para valorar el aporte nutricional del alimento. Objetivo. Determinar el contenido de $\mathrm{Ca}, \mathrm{P}, \mathrm{K}, \mathrm{Na}, \mathrm{Mg}, \mathrm{S}, \mathrm{Cu}, \mathrm{I}, \mathrm{Fe}, \mathrm{Mn}$ y Zn en 34 alimentos importados para perros, gatos, conejos, hámsters, peces ornamentales y tortugas, y comparar los resultados con las recomendaciones encontradas en la literatura. Materiales y métodos. Durante los meses de agosto y diciembre del año 2018, se obtuvieron diez muestras de alimentos para perros y gatos, cinco para peces ornamentales, cuatro para tortugas, tres para hámster y dos para conejos. Estas se adquirieron de manera directa en diferentes puntos de venta en la Gran Área Metropolitana, San José, Costa Rica. Las muestras se analizaron en el Centro de Investigación en Nutrición Animal de la Universidad de Costa Rica. Se cuantificaron los minerales $\mathrm{Ca}, \mathrm{P}, \mathrm{K}, \mathrm{Mg}, \mathrm{S}, \mathrm{Cu}, \mathrm{I}, \mathrm{Fe}, \mathrm{Mn}$, y Zn con base en la metodología recomendada por la Association of Official Analytical Chemists. Se calculó el contenido promedio por grupo de alimento según la especie animal, la desviación estándar, valores máximo y mínimo; se comparó con las recomendaciones nutricionales para minerales encontradas en la literatura. Resultados. Los alimentos estudiados cumplieron, en promedio, con los requerimientos minerales de los animales de compañía. Se observaron desbalances en el contenido de $\mathrm{Ca}, \mathrm{P}$ y $\mathrm{Mg}$ para el caso de alimentos para tortugas, altos contenidos de yodo en alimentos para perros y relaciones desbalanceadas entre minerales. Conclusiones. Se generó una base de datos con valores promedios y su dispersión en once minerales en 34 muestras de alimentos comerciales de siete animales de compañía en Costa Rica. Se observó la poca información sobre límites superiores recomendados y requerimientos en especies como hámster, peces ornamentales o tortugas.

Palabras clave: animales de compañía, contenido mineral, deficiencias minerales, interacción entre nutrientes, nutrición animal.

\begin{abstract}
Introduction. The mineral content in pet food is not always displayed on the label. It is important to know this information in order to assess the nutritional contribution of the food. Objective. To determine the content of Ca, $\mathrm{P}, \mathrm{K}$, $\mathrm{Na}, \mathrm{Mg}, \mathrm{S}, \mathrm{Cu}, \mathrm{I}, \mathrm{Fe}, \mathrm{Mn}$, and $\mathrm{Zn}$ in 34 imported foods for dogs, cats, rabbits, hamsters, ornamental fish, and turtles, and to compare the results with the recommendations found in the literature. Materials and methods. During the
\end{abstract}


months of August and December of 2018, ten samples were collected of dog and cat food, five for ornamental fishes, four for turtles, three for hamsters, and two for rabbits. These were acquired directly at different points of sale in the Greater Metropolitan Area, San Jose, Costa Rica. The samples were analyzed at the Animal Nutrition Research Center of the Universidad de Costa Rica. The minerals $\mathrm{Ca}, \mathrm{P}, \mathrm{K}, \mathrm{Mg}, \mathrm{S}, \mathrm{Cu}, \mathrm{I}, \mathrm{Fe}, \mathrm{Mn}$, and Zn were quantified based on the methodology recommended by the Association of Official Analytical Chemists. The average content per feed group according to the animal species, standard deviation, maximum and minimum values were calculated compared with the nutritional recommendations for minerals found in the literature. Results. The foods studied met, on average, the mineral requirements for pets. Imbalances in $\mathrm{Ca}, \mathrm{P}$, and $\mathrm{Mg}$ content in the turtle foods, high iodine contents in dog foods, and unbalanced mineral relations were observed. Conclusions. A database was generated with average values and their dispersion in eleven minerals in 34 samples of commercial foods for seven companion animals in Costa Rica. Little information was available regarding the recommended upper limits and requirements in species such as hamsters, ornamental fish or turtles.

Keywords: pets, mineral content, mineral content, mineral deficiencies, nutrient interaction, animal nutrition.

\section{Introducción}

Las mascotas son importantes para sus dueños, existe entre ambos una recíproca muestra de afecto, lealtad y acompañamiento (Gómez et al., 2007). En Costa Rica, según la Ley 7451 de Bienestar de los Animales, el cuidado de las mascotas debe incluir un ambiente adecuado, tiempo para compartir, por ejemplo, caminatas con el perro (Canis lupus familiaris) y juegos con los gatos (Felis catus), y una apropiada dieta y manejo sanitario (Asamblea Legislativa de la República de Costa Rica, 1994).

Al profundizar en el manejo alimenticio de los animales de compañía, estos presentan requerimientos nutricionales de energía, proteína (aminoácidos esenciales), fibra, grasa (ácidos grasos esenciales) y minerales, según su peso, estado fisiológico y grado de actividad (National Research Council, 2006).

Los minerales constituyen una parte fundamental de la nutrición, al estar involucrados en el metabolismo celular (Fuller, 2004). En función de la cantidad necesaria que se aporta a través de la dieta, estos se dividen en dos grupos: macrominerales (Ca, P, K, Na Mg y Cl) y microminerales (Fe, Zn, Mn, Cu, I y Se) (Case et al., 1997).

Los minerales constituyen entre 4 y $5 \%$ del peso vivo de los animales, por lo que un desbalance (exceso o deficiencia) podría promover el desarrollo de enfermedades metabólicas (Campos-Granados, 2015), por lo que se asocia negativamente con la salud (Davies et al., 2017). Un adecuado aporte mineral proveniente de la dieta es necesario para mantener una buena salud, ya que estos participan en los sistemas inmune, reproductivo y nervioso, así como en los procesos de crecimiento y digestivos (National Research Council, 2006). Desbalances en el contenido de la dieta de $\mathrm{Ca}, \mathrm{P}, \mathrm{Zn}, \mathrm{Fe}, \mathrm{Cu}, \mathrm{Mn}$ y $\mathrm{Se}$, se han asociado con la aparición de enfermedades nutricionales en mascotas (Gagné et al., 2013).

El exceso o deficiencia de un mineral puede afectar la utilización en el organismo de otros minerales, por lo que conocer el contenido de estos elementos en los alimentos comerciales o elaborados en casa, permite que el profesional en nutrición animal brinde una dieta balanceada de acuerdo con los requerimientos de cada especie (Remillard, 2008).

La deficiencia leve de K no presenta signos clínicos; sin embargo, si el desbalance es persistente puede derivar en hipopotasemia y mal funcionamiento de los riñones en perros (Case et al., 1997; Hand et al., 2000; National Research Council, 2006). En cambio, el exceso en la dieta $(>1 \%)$ aumenta el riesgo de nefritis y reduce la ingesta y la absorción de Mg. 
En conejos (Oryctolagus cuniculus), más de $4 \%$ de Ca en la dieta aumenta el riesgo de urolitiasis, calcificación de tejidos blandos y deficiencia en la absorción de P. El exceso de P en la dieta ( $>0,9 \%)$, puede disminuir el consumo y la fertilidad en las hembras. La deficiencia de $\mathrm{Mg}$ en conejos es la responsable de cuadros de alopecia, nerviosismo, pelaje hirsuto y tricofagia; el exceso del ion $\mathrm{Na}^{+}$en forma de sal $(>1 \%)$, reduce el crecimiento. El déficit de $\mathrm{Cu}$ ocasiona crecimiento retardado, pelaje gris y deformaciones óseas; el de Mn provoca problemas en las patas, por una mala consistencia de los huesos; mientras que el yodo provoca la aparición de bocio (de Blas \& Wiseman, 2010; National Research Council, 1977; Ricci et al., 2010).

En peces ornamentales la información sobre la nutrición mineral es escasa, se menciona que va a depender del ambiente, la edad, la especie y la tasa de crecimiento (Lall \& Lewis-McCrea, 2007; Lim \& Klesius, 2019). Cubrir los requerimientos de $\mathrm{P}$ es importante para los peces en desarrollo, debido a que este mineral interviene en la mineralización del esqueleto (Lall \& Lewis-McCrea, 2007). Se ha observado que un desbalance de macrominerales en la dieta causa deficiencias o toxicidad en peces ornamentales; niveles inferiores a $30 \mathrm{ppm}$ de Fe causa anemia, anorexia y crecimiento deficiente, mientras que el exceso $(>175 \mathrm{ppm})$ puede incrementar la susceptibilidad a infecciones. La deficiencia de $\mathrm{Zn}(<15 \mathrm{ppm})$ se asocia a un pobre crecimiento, anorexia, cataratas y alta mortalidad (Lim \& Klesius, 2000).

En Costa Rica, al mes de abril del año 2021, se encontraban registrados como alimentos balanceados en la Dirección de Alimentos para Animales, más de 712 productos. Estos se pueden clasificar según su especie de destino en: gatos (43\%), perros cachorros (30\%), peces ornamentales (24\%), tortugas (Trachemy sp) (2\%), conejos $(1,5 \%)$ y hámster (Mesocricetus auratus) $(0,4 \%)$ (Ministerio de Agricultura y Ganadería, 2021). Los lineamientos para el correcto etiquetado de los alimentos para animales están dados por el "Reglamento Técnico Centroamericano, RTCA 65.05.52:11: Productos utilizados en alimentación animal y establecimientos requisitos de registro sanitario y control" (Ministerio de Comercio Exterior et al., 2012).

Entre los componentes, cuyo contenido se debe de garantizar en el etiquetado de un alimento balanceado, se encuentran: humedad (\% máximo), proteína (\% mínimo), grasa o extracto etéreo (\% mínimo), fibra (\% máximo), energía (contenido mínimo calculado), sal (\% mínimo y máximo), calcio (\%) y fósforo (\% mínimo) (Ministerio de Comercio Exterior et al., 2012). De la normativa no se deriva una obligatoriedad de informar en la etiqueta sobre el aporte mineral de $\mathrm{K}, \mathrm{Na}, \mathrm{Mg}, \mathrm{Cl}, \mathrm{Fe}, \mathrm{Zn}, \mathrm{Mn}, \mathrm{Cu}, \mathrm{I}$ y $\mathrm{Se}$.

El conocer el contenido mineral del alimento es importante, pero tiene que acompañarse de la información del consumo $\left(\mathrm{kg} \mathrm{día}^{-1}\right)$ que presentan los animales, análisis de biodisponibilidad y considerar las variaciones en los resultados asociados al método analítico utilizado para el análisis (Hendriks et al., 2015), con la intención de descartar problemas de deficiencias minerales en los animales de compañía. El desbalance se puede generar como una consecuencia de una menor cantidad de mineral presente en el alimento consumido o por interacciones antagónicas entre minerales que afecta la absorción y utilización del que se encuentra en menor concentración.

El objetivo de este trabajo fue determinar el contenido de $\mathrm{Ca}, \mathrm{P}, \mathrm{K}, \mathrm{Na}, \mathrm{Mg}, \mathrm{S}, \mathrm{Cu}, \mathrm{I}, \mathrm{Fe}, \mathrm{Mn}$ y $\mathrm{Zn}$ presentes en 34 alimentos importados para perros, gatos, conejos, hámster, peces ornamentales, tortugas y comparar los resultados con las recomendaciones encontradas en la literatura.

\section{Materiales y Métodos}

\section{Obtención de las muestras de alimento para mascotas}

Entre los meses de agosto y diciembre del año 2018, se obtuvieron 34 muestras de alimentos balanceados importados, mediante compra directa en diferentes establecimientos, ubicados en el Gran Área Metropolitana de la provincia de San José, Costa Rica. Previo a la compra, se realizó una búsqueda en los registros activos de alimentos 
balanceados de la Dirección de Alimentos para Animales, que cumplieran el requisito de ser elaborado en otro país. De esta preselección, se obtuvieron diez muestras de alimento para perro (Canis lupus familiaris) [cinco de animal adulto (1460 g) y cinco para cachorro (1600 g)], diez muestras de alimento para gato (Felis catus) [(cinco de adulto (1906 g) y cinco de cachorro (1460 g)], cinco muestras para peces ornamentales (Carassius sp. y Betta sp.) (69 g), cuatro para tortugas (Trachemy sp.) (80 g), tres para mantenimiento de hámster (Mesocricetus auratus $(517 \mathrm{~g}) \mathrm{y}$ dos para mantenimiento de conejos (Oryctolagus cuniculus) (2270 g).

\section{Tratamiento y análisis químicos de las muestras}

Posterior a la compra de las muestras de los alimentos balanceados importados, estas se trasladaron en su empaque comercial al Centro de Investigación en Nutrición Animal (CINA) de la Universidad de Costa Rica, ubicado en San Pedro de Montes de Oca, San José, Costa Rica. Las muestras se secaron por tres días a $60{ }^{\circ} \mathrm{C}$, posterior a esta etapa, se molieron a $1 \mathrm{~mm}$ de grosor, se colocó $5 \mathrm{~g}$ de muestra en un crisol de porcelana y se incineraron a $550{ }^{\circ} \mathrm{C}$ por $2 \mathrm{~h}$. Las cenizas obtenidas se analizaron según el método correspondiente para Ca (968.08), P (965.17), Mg (968.08), Na (985.35), K (985.35), S (924.06), Cu (968.08), Fe (968.08), I (934.02), Mn (968.08) y Zn (968.08) (Association of Analytical Communities International, 2005).

\section{Análisis de la información}

Los datos obtenidos se utilizaron en un modelo estadístico de tipo descriptivo, para obtener valores de medidas de tendencia central como el promedio, la desviación estándar, valores mínimo y máximo (Llínas-Solano \& Rojas-Álvares, 2005). Los resultados obtenidos para el contenido medio de cada mineral, se compararon con los valores recomendados según Chen et al. (2014), Corcoran y Roberts-Sweeney (2014), de Blas y Wiseman (2010), Donoghue y McKeown (1999), Hand et al. (2000), Mulder (2012), National Research Council (1977; 1995; 2006), Rawski et al. (2018) y Velasco-Santamaría y Corredor-Santamaría (2011), para cada animal de compañía evaluado (Cuadro 1).

\section{Resultados}

\section{Perro adulto y cachorro}

En los alimentos para perros adultos (Cuadro 2), se observó que el contenido promedio de macro y micro minerales de los alimentos importados, satisface el requerimiento mínimo sugerido (Cuadro 1). Al analizar de forma individual las muestras (datos no publicados), se observó para cada mineral, que su aporte fue superior al requerimiento nutricional mínimo. Se encontraron valores superiores al máximo recomendado para yodo en alimentos para perro adulto (4 ppm).

Los alimentos para perro cachorro contenían, en promedio, mayor cantidad que la sugerida en el requerimiento mínimo (Cuadro 1). De forma individual, la mayoría de las muestras para perro cachorro analizadas también cumplían con los requerimientos minerales, a excepción de un alimento con $-0,3 \%$ de $\mathrm{P}$ y otro con $-1,18 \mathrm{ppm}$ de $\mathrm{Cu}$, que los requerimientos mínimos. Dos muestras presentaron una concentración de $\mathrm{Ca}$ de $+0,5 \%$ que la recomendación máxima del requerimiento de calcio (National Research Council, 2006). 
Cuadro 1. Niveles de macro y micro minerales recomendados en la literatura para la nutrición de perros (Canis lupus familiaris), gatos (Felis catus), hámster (Mesocricetus auratus), tortugas (Trachemy sp.), conejos (Oryctolagus cuniculus) y peces ornamentales (Carassius sp. y Betta sp.). San José, Costa Rica. 2021.

Table 1. Levels of macro and micro minerals recommended in the literature for the nutrition of dogs (C. lupus familiaris), cats $(F$. catus), hamsters (M. auratus), turtles (Trachemy sp.), rabbits (O. cuniculus) and ornamental fish (Carassius sp. y Betta sp.). San José Costa Rica. 2021.

\begin{tabular}{|c|c|c|c|c|c|c|c|c|}
\hline \multirow[t]{2}{*}{ Mineral } & \multicolumn{2}{|c|}{ Perro $^{\Omega}$} & \multicolumn{2}{|c|}{ Gato $^{2}$} & \multirow[t]{2}{*}{ Hámster* } & \multirow[t]{2}{*}{ Conejo $^{\infty}$} & \multirow{2}{*}{$\begin{array}{l}\text { Peces orna- } \\
\text { mentales }\end{array}$} & \multirow[t]{2}{*}{ Tortugas } \\
\hline & Adulto & Cachorro & Adulto & Cachorro & & & & \\
\hline $\mathrm{Ca}(\%)$ & 0,2 & 0,8 & 0,16 & 0,52 & 0,47 & 0,4 & - & 5,7 \\
\hline $\mathrm{P}(\%)$ & 0,3 & 1 & 0,14 & 0,48 & 0,2 & 0,22 & $0,3^{\circ}$ & 3 \\
\hline $\operatorname{Mg}(\%)$ & 0,018 & 0,018 & 0,02 & 0,016 & 0,06 & 0,04 & $0,06^{\wedge}$ & 0,97 \\
\hline $\mathrm{Na}(\%)$ & 0,03 & 0,22 & 0,065 & 0,124 & 0,15 & 0,2 & - & - \\
\hline $\mathrm{K}(\%)$ & 0,4 & 0,44 & 0,52 & 0,268 & 0,6 & 0,6 & - & - \\
\hline S (\%) & - & - & - & - & - & - & - & - \\
\hline $\mathrm{Cu}(\mathrm{ppm})$ & 6 & 11 & 5 & 4,5 & 1,6 & 3 & - & 4 \\
\hline $\mathrm{Fe}(\mathrm{ppm})$ & 30 & 72 & 80 & 70 & 140 & - & $80^{+}$ & 266 \\
\hline I (ppm) & 0,7 & 0,21 & 1,3 & 0,12 & 0,15 & 0,2 & - & - \\
\hline $\mathrm{Mn}$ (ppm) & 4,8 & 5,6 & 4,8 & 4,8 & 3,6 & 2,5 & - & - \\
\hline $\mathrm{Zn}(\mathrm{ppm})$ & 60 & 40 & 74 & 50 & 9,2 & 25 & $100^{+}$ & 35 \\
\hline
\end{tabular}

Fuente / Source: $\infty$ de Blas \& Wiseman (2010), ${ }^{\odot}$ Corcoran \& Roberts-Sweeney (2014), * Mulder (2012); National Research Council (1995), ${ }^{\Omega}$ National Research Council (2006), Rawski et al. (2018), + Sales \& Janssens (2003), ^ Velasco-Santamaría \& CorredorSantamaría (2011).

\section{Gato adulto y cachorro}

Los alimentos para gato adulto y para gato cachorro presentaron un contenido mineral que se encontró entre los niveles recomendados (National Research Council, 2006) (Cuadros 1 y 2), a excepción de uno para gato adulto, que contenía $-0,09 \%$ de $\mathrm{K}$.

\section{Hámster}

Los niveles de minerales (Cuadros 1 y 2) de las muestras de alimento para hámster (valores promedio e individuales) se encontraron por encima de los requerimientos mínimos indicados por Mulder (2012) y National Research Council (1995). Excepto en el contenido para Na y K, donde para cada mineral se obtuvo un alimento que no cumplió con la concentración mínima recomendada.

\section{Conejo}

Los alimentos para conejos, tanto de forma individual como promedio, presentaron para los once elementos analizados, un contenido de nutrientes superior al requerimiento mínimo sugerido por el National Research Council (1977) y de Blas y Wiseman (2010) (Cuadro 1). 
Cuadro 2. Contenido mineral de alimentos para perros (Canis lupus familiaris), gatos (Felis catus), hámster (Mesocricetus auratus), tortugas (Trachemy sp.), conejos (Oryctolagus cuniculos) y peces ornamentales (Carassius sp. y Betta sp.), analizados en el Centro de Investigación en Nutrición Animal de la Universidad de Costa Rica, e importados y comercializados en San José, Costa Rica, 2020.

Table 2. Mineral content of food analyzed in the Animal Nutrition Research Center of the University of Costa Rica for dogs (C. lupus familiaris), cats (F. catus), hamsters (M. auratus), turtles (Trachemy sp.), rabbits (O. cuniculos), and ornamental fish (Carassius sp. y Betta sp.), imported and marketed in San José, Costa Rica, 2020.

\begin{tabular}{|c|c|c|c|c|c|c|c|c|c|c|c|c|}
\hline Alimento & Elemento* & $\mathrm{Ca}(\%)$ & $P(\%)$ & $\begin{array}{l}\mathrm{Mg} \\
(\%)\end{array}$ & $\mathrm{Na}(\%)$ & $\mathbf{K}(\%)$ & $S(\%)$ & $\underset{(\mathbf{p p m})}{\mathrm{Cu}}$ & $\begin{array}{c}\mathbf{F e} \\
(\mathbf{p p m})\end{array}$ & I (ppm) & $\begin{array}{c}\text { Mn } \\
\text { (ppm) }\end{array}$ & $\begin{array}{c}\mathbf{Z n} \\
(\mathbf{p p m})\end{array}$ \\
\hline \multirow{4}{*}{$\begin{array}{l}\text { Perro } \\
\text { adulto } \\
(n=5)\end{array}$} & $\mathrm{m}$ & 0,77 & 0,58 & 0,10 & 0,31 & 0,51 & 0,21 & 9,12 & 140,10 & 541,60 & 22,18 & 141,25 \\
\hline & M & 1,37 & 1,33 & 0,16 & 0,48 & 0,79 & 0,30 & 13,06 & 274,47 & 1027,50 & 64,12 & 243,51 \\
\hline & $\mathbf{X}$ & 1,02 & 0,84 & 0,13 & 0,39 & 0,67 & 0,25 & 11,36 & 220,71 & 704,78 & 32,24 & 201,06 \\
\hline & $\mathbf{s}$ & 0,24 & 0,29 & $\mathbf{0 , 0 3}$ & $\mathbf{0 , 0 7}$ & $\mathbf{0 , 1 1}$ & 0,04 & 1,62 & 49 & 194 & 18 & 46 \\
\hline \multirow{4}{*}{$\begin{array}{l}\text { Perro } \\
\text { cachorro } \\
(n=5)\end{array}$} & $\mathrm{m}$ & 1,16 & 0,70 & 0,00 & 0,36 & 0,50 & 0,19 & 9,82 & 246,29 & 531,00 & 27,31 & 164,50 \\
\hline & M & 2,31 & 1,56 & 0,17 & 0,72 & 0,86 & 0,23 & 94,73 & 511,00 & 1714,30 & 63,61 & 1471,72 \\
\hline & $\mathbf{X}$ & 1,62 & 1,15 & 0,12 & $\mathbf{0 , 5 1}$ & 0,68 & 0,21 & 30,12 & 378,09 & 942,20 & 43,33 & 476,06 \\
\hline & $\mathbf{s}$ & 0,52 & 0,32 & $\mathbf{0 , 0 7}$ & 0,14 & 0,15 & 0,02 & 36 & 104 & 485 & 15 & 557 \\
\hline \multirow{4}{*}{$\begin{array}{l}\text { Gato } \\
\text { adulto } \\
(n=5)\end{array}$} & $\mathrm{m}$ & 0,88 & 0,69 & 0,09 & 0,44 & 0,43 & 0,10 & 8,54 & 167,45 & 170,30 & 15,80 & 100,65 \\
\hline & M & 1,84 & 1,01 & 0,17 & 0,77 & 0,88 & 0,16 & 14,49 & 466,63 & 412,10 & 80,36 & 238,88 \\
\hline & $\mathbf{X}$ & 1,32 & 0,88 & 0,14 & 0,53 & 0,68 & 0,13 & 10,68 & 268,87 & 308,24 & 48,08 & 184,63 \\
\hline & $\mathbf{s}$ & $\mathbf{0 , 3 7}$ & 0,13 & $\mathbf{0 , 0 3}$ & 0,14 & $\mathbf{0 , 1 8}$ & 0,02 & 2,38 & 127 & 97 & 28 & 58 \\
\hline \multirow{4}{*}{$\begin{array}{l}\text { Gato } \\
\text { cachorro } \\
(n=5)\end{array}$} & $\mathrm{m}$ & 1,17 & 0,81 & 0,11 & 0,37 & 0,34 & 0,07 & 8,06 & 194,31 & 63,50 & 15,54 & 112,49 \\
\hline & M & 1,87 & 1,41 & 0,17 & 0,55 & 0,72 & 0,13 & 18,61 & 3165,55 & 506,90 & 64,60 & 373,54 \\
\hline & $\mathbf{X}$ & 1,51 & 1,00 & 0,14 & 0,50 & 0,58 & 0,10 & 13,80 & 809,31 & 294,64 & 44,30 & 209,49 \\
\hline & $\mathbf{s}$ & $\mathbf{0 , 3 1}$ & 0,25 & $\mathbf{0 , 0 3}$ & $\mathbf{0 , 0 7}$ & 0,15 & 0,02 & 3,88 & 48,8 & 165 & 19,6 & 99,7 \\
\hline \multirow{4}{*}{$\begin{array}{l}\text { Hámster } \\
(\mathrm{n}=3)\end{array}$} & $\mathrm{m}$ & 0,43 & 0,40 & 0,18 & 0,08 & 0,62 & 0,04 & 5,43 & 112,23 & 548,00 & 40,83 & 43,22 \\
\hline & M & 1,52 & 0,77 & 0,36 & 0,28 & 1,15 & 0,10 & 15,42 & 1335,21 & 692,60 & 163,67 & 255,44 \\
\hline & $\mathbf{X}$ & 0,98 & 0,56 & 0,26 & 0,19 & 0,97 & $\mathbf{0 , 0 7}$ & 11,51 & $\mathbf{5 7 7 , 9 5}$ & 603,33 & 103,58 & 132,74 \\
\hline & $\mathbf{s}$ & 0,55 & 0,19 & 0,09 & 0,10 & $\mathbf{0 , 3 0}$ & $\mathbf{0 , 0 3}$ & 5,34 & 661 & 78 & 61 & 110 \\
\hline \multirow{4}{*}{$\begin{array}{l}\text { Conejo } \\
(\mathrm{n}=2)\end{array}$} & $\mathrm{m}$ & 0,67 & 0,32 & 0,25 & 0,22 & 0,77 & 0,05 & 198,76 & 331,48 & 557,80 & 66,97 & 58,64 \\
\hline & M & 0,69 & 0,47 & 0,28 & 0,23 & 0,79 & 0,07 & 222,05 & 425,77 & 585,70 & 82,02 & 63,57 \\
\hline & $\mathbf{X}$ & 0,68 & 0,40 & 0,27 & 0,23 & $\mathbf{0 , 7 8}$ & 0,06 & 210,41 & 378,63 & 571,75 & 74,50 & 61,11 \\
\hline & $\mathbf{s}$ & $\mathbf{0 , 0 1}$ & $\mathbf{0 , 1 1}$ & $\mathbf{0 , 0 2}$ & $\mathbf{0 , 0 1}$ & $\mathbf{0 , 0 1}$ & $\mathbf{0 , 0 1}$ & 16,5 & 66,7 & 19,7 & 10,6 & 3,49 \\
\hline \multirow{4}{*}{$\begin{array}{l}\text { Peces orna- } \\
\text { mentales } \\
(\mathrm{n}=5)\end{array}$} & $\mathrm{m}$ & 1,40 & 1,01 & 0,17 & 0,30 & 0,47 & 0,05 & 0,87 & 207,31 & 377,20 & 34,68 & 14,86 \\
\hline & M & 2,47 & 1,79 & 0,27 & 0,77 & 0,83 & 0,08 & 11,28 & 2602,83 & 722,10 & 68,36 & 95,31 \\
\hline & $\mathbf{X}$ & 1,80 & 1,28 & 0,22 & $\mathbf{0 , 5 3}$ & 0,67 & 0,06 & 5,28 & 737,15 & 515,86 & 49,10 & 49,07 \\
\hline & $\mathbf{s}$ & 0,43 & $\mathbf{0 , 3 1}$ & 0,04 & 0,18 & 0,14 & 0,01 & 4,30 & 1045 & 149 & 13,74 & 36,31 \\
\hline \multirow{4}{*}{$\begin{array}{l}\text { Tortuga } \\
(\mathrm{n}=4)\end{array}$} & $\mathrm{m}$ & 0,89 & 0,74 & 0,15 & 0,15 & 0,34 & 0,00 & 2,84 & 136,30 & 422,40 & 41,50 & 87,17 \\
\hline & M & 6,06 & 1,45 & 0,35 & 0,59 & 0,59 & 0,08 & 95,91 & 719,31 & 592,10 & 169,48 & 125,81 \\
\hline & $\mathbf{X}$ & 2,70 & 1,01 & 0,21 & 0,38 & 0,42 & 0,05 & 43,16 & 410,42 & 510,00 & 93,13 & 102,99 \\
\hline & $\mathbf{s}$ & 2,39 & 0,32 & 0,09 & 0,18 & 0,12 & $\mathbf{0 , 0 3}$ & 38,7 & 239 & 79,2 & 54,4 & 20,2 \\
\hline
\end{tabular}

* m: mínimo, M: máximo, X: promedio, s: desviación estándar. / * m: mínimum, M: maximum, X: average, s: standard deviation.

\section{Peces ornamentales}

Las muestras de alimentos para peces ornamentales (Cuadro 1 y 2) cumplieron con los requerimientos minerales sugeridos en la literatura por Corcoran y Roberts-Sweeney (2014) y Velasco-Santamaría y CorredorSantamaría (2011). 


\section{Tortugas}

Las muestras de alimentos para tortuga presentaron menor contenido de $\mathrm{Ca}$ y $\mathrm{P}$ que el recomendado por Donoghue y McKeown (1999); Hand et al. (2000) y Rawski et al. (2018). Los niveles de Mg también fueron inferiores a la recomendación mínima. Además, se encontró un alimento con un contenido menor de $\mathrm{Cu}$ que la recomendación mínima, descrita por Chen et al. (2014) y Rawski et al. (2018).

\section{Relaciones entre minerales}

Se determinaron variaciones asociadas a la relación evaluada y al tipo de alimento, donde se obtuvieron valores superiores o inferiores a lo ideal, según lo calculado con la información de los requerimientos minerales de cada especie (Cuadro 3). En ambos casos, los desbalances podrían afectar la absorción y utilización del elemento que se encuentre en menor cantidad, en los alimentos balanceados analizados.

Cuadro 3. Relación entre minerales en alimentos para perros (Canis lupus familiaris), gatos (Felis catus), hámster (Mesocricetus auratus), tortugas (Trachemy sp.), conejos (Oryctolagus cuniculus) y peces ornamentales (Carassius sp. y Betta sp.), analizados en el Centro de Investigación en Nutrición Animal de la Universidad de Costa Rica, e importados y comercializados en San José, Costa Rica. 2021.

Table 3. Ratio between minerals in imported and marketed in San Jose of food for dogs (C. lupus familiaris), cats (F. catus), hamsters (M. auratus), turtles (Trachemy sp.), rabbits (O. cuniculus), and ornamental fish (Carassius sp. y Betta sp.), analyzed in Animal Nutrition Research Center of the University of Costa Rica. San José, Costa Rica. 2021.

\begin{tabular}{lccccccccccc}
\hline & \multicolumn{1}{c}{ Relaciones entre minerales } \\
\cline { 2 - 12 } Especie & \multicolumn{2}{c}{ Zn:Cu } & \multicolumn{2}{c}{ Fe:Cu } & \multicolumn{2}{c}{ K:Mg } & \multicolumn{2}{c}{ Zn:Mn } & \multicolumn{2}{c}{ K:Na } \\
\cline { 2 - 12 } & Real & Ideal* & Real & Ideal & Real & Ideal & Real & Ideal & Real & Ideal \\
\hline Perro adulto & 17,70 & 10 & 19,43 & 5 & 5,15 & 22,2 & 6,24 & 12,5 & 1,72 & 13,3 \\
Perro cachorro & 15,81 & 3,6 & 12,55 & 6,5 & 5,67 & 24,4 & 10,99 & 7,1 & 1,33 & 2 \\
Gato adulto & 17,29 & 14,8 & 25,18 & 16 & 4,86 & 26 & 3,84 & 15,4 & 1,28 & 8 \\
Gato cachorro & 15,18 & 11,1 & 58,65 & 31,1 & 4,14 & 16,7 & 4,73 & 10,4 & 1,16 & 2,2 \\
Hámster & 11,53 & 5,7 & 50,21 & 87,5 & 3,73 & 10 & 1,28 & 2,6 & 5,11 & 4 \\
Conejo & 0,29 & 8,3 & 1,80 & - & 2,89 & 15 & 0,82 & 10 & 3,39 & 3 \\
Peces ornamentales & 9,29 & - & 139,61 & - & 3,05 & - & 1,00 & - & 1,26 & - \\
Tortuga & 2,39 & 8,7 & 9,51 & 66,5 & 2,00 & - & 1,11 & - & 1,11 & - \\
\hline
\end{tabular}

*Relación mineral obtenida del requerimiento diario para cada mineral. / Mineral ratio obtained from daily requirement for each mineral.

\section{Discusión}

Al comparar el contenido mineral de los alimentos analizados, se observó que, en general, cumplieron con las recomendaciones nutricionales. Sin embargo, los alimentos para perro cachorro con menor contenido de P podrían propiciar la aparición de enfermedades esqueléticas, al ser un constituyente de moléculas, como los fosfolípidos, numerosas enzimas, adenosín trifosfato (ATP) y, junto con el Ca, forma la hidroxiapatita, constituyente fundamental de los huesos. Además, el P participa en el metabolismo de grasas, proteínas y carbohidratos (Case et al., 1997). 
Las concentraciones de P y Ca en la dieta tienen un efecto directo en la mineralización ósea (Lauten et al., 2002), aspecto de suma importancia en los animales que se encuentran en la etapa de desarrollo.

La concentración de $\mathrm{K}$ en los alimentos analizados presentó niveles apropiados según los requerimientos establecidos para cada especie, pero se debe prestar atención en las relaciones $\mathrm{K}$ / Mg y K / Na, donde los valores obtenidos podrían provocar antagonismo con estos minerales, debido a que se encuentran por encima o por debajo de las relaciones ideales, donde estos cationes compiten por los puntos de absorción a nivel intestinal (Goff, 2018). Para el $\mathrm{Cu}$, las relaciones obtenidas con Zn y Fe podrían generar antagonismos en su absorción, lo que podría provocar estados de anemia en los animales de compañía, retraso del crecimiento, lesiones óseas y trastornos neuromusculares, por su participación en procesos metabólicos del animal (oxidasas) (National Research Council, 2006).

Para el Mg, Fe, Mn, y Zn, no se encontraron deficiencias, por lo que la participación de estos minerales en procesos metabólicos (transporte de oxígeno $(\mathrm{Fe})$, presencia de hígado graso $(\mathrm{Mn})$, metabolismo de ácidos grasos y carbohidratos (Zn)), reproductivos (Mn y Zn), salud de la piel (Zn) y de crecimiento (Zn y Mn) de la mascota, no se verán afectados, como el caso del $\mathrm{Mg}$, el cual se asocia como constituyente de los huesos y líquidos intracelulares, metabolismo de carbohidratos y lípidos, y componente activo de enzimas (Case et al., 1997; Hand et al., 2000).

En los alimentos para hámster, al comparar los valores promedios e individuales de las muestras con el requerimiento mínimo (Mulder, 2012; National Research Council, 1995), sí cumplieron con estas recomendaciones; excepto para $\mathrm{Na}$ y el $\mathrm{K}$, en cada caso, un alimento no cumplió con la concentración mínima recomendada. En hámster no se han descrito signos de la deficiencia de estos minerales; sin embargo, en ratas, la deficiencia de $\mathrm{Na}$ se asocia a un inferior crecimiento, lesiones en las corneas y huesos frágiles; mientras que la de $\mathrm{K}$ se asocia con reducción de ingesta y del crecimiento, cianosis, diarrea y ascitis (National Research Council, 1995).

En los alimentos para tortuga se observó que las muestras presentaron un contenido de $\mathrm{Ca}$ y $\mathrm{P}$ inferior al recomendado. Estos minerales son de gran importancia en estos animales, por su mayor proporción esquelética que se traduce en huesos, caparazón y plastrón frágiles y deformados. La enfermedad ósea metabólica es causada por deficiencias en Ca, P y vitamina D (Donoghue, 1998; Hand et al., 2000; Rawski et al., 2018). Los niveles de Mg también fueron inferiores a la recomendación mínima, factor nutricional que podría comprometer la salud esquelética de las tortugas, ya que el $\mathrm{Mg}$ está relacionado en el metabolismo del Ca y en la constitución de los huesos (Donoghue \& McKeown, 1999). Si los alimentos analizados se utilizaran como única fuente de nutrientes, estos podrían propiciar la aparición de alteraciones esqueléticas en las tortugas, por su participación en la mineralización de los huesos, el buen estado del tejido cutáneo y su metabolismo en general (Chen et al., 2014; Rawski et al., 2018).

Además de analizar las cantidades mínimas necesarias para no causar deficiencias, es importante verificar que no se presenten excesos de minerales, según el límite superior recomendado. Esto se puede apreciar en las relaciones entre minerales evaluadas en este trabajo, donde un exceso de un mineral afecta o favorece la utilización de otro (interacciones antagónicas o sinérgicas). Estas interacciones, producto a las relaciones entre minerales determinadas en este trabajo, pueden presentarse durante la digestión del alimento, en el tracto digestivo por los mecanismos de absorción o asimilación de la célula, sitios de almacenamiento o inhibición de la actividad enzimática, durante el transporte o en la vía excretora (Hand et al., 2000). Aunque el alimento contenga las concentraciones minerales adecuadas, si se sub-dosifica en la ración, el aporte real de uno o varios minerales, puede ser menor al requerimiento mínimo (Hand et al., 2000). Esta situación es más relevante en animales de menor peso y talla, debido a que la cantidad de alimento a consumir es menor.

Se encontraron altos contenidos de yodo en alimentos para perro adulto, superior al contenido máximo sugerido (4 ppm). Es importante considerar, que este mineral es el componente principal de las hormonas tiroideas, que se encargan entre otras funciones de regular el ritmo metabólico; en animales adultos, los signos de exceso de yodo son lagrimeo, salivación y descarga nasal excesiva, pelaje seco y quebradizo (National Research Council, 
2006). De los alimentos para perro cachorro, dos alimentos tuvieron $+0,5 \%$ de Ca, cuyo exceso en animales de raza grande producen anormalidades esqueléticas, por ejemplo osteocondrosis (National Research Council, 2006; Tal et al., 2018).

En los alimentos para peces ornamentales, el mineral que se debe suplementar es el $\mathrm{P}$, debido a que los demás elementos se encuentran en niveles aceptables y pueden ser absorbidos del medio a través de las agallas (Corcoran \& Roberts-Sweeney, 2014; Velasco-Santamaría \& Corredor-Santamaría, 2011). En el caso del hierro, aportes por encima de 175 ppm de Fe, puede aumentar la susceptibilidad a infecciones (Lim \& Klesius, 2000).

\section{Conclusiones}

Este trabajo describe el contenido de $\mathrm{K}, \mathrm{Na}, \mathrm{Mg}, \mathrm{S}, \mathrm{Cu}, \mathrm{Fe}, \mathrm{I}, \mathrm{Mn}$ y $\mathrm{Zn}$ de alimentos para mascotas, por lo que genera información valiosa para cumplir con los requerimientos nutricionales en el desarrollo de programas nutricionales para perros, gatos, conejos, hámster, tortugas y peces ornamentales.

El perfil mineral de los alimentos evaluados presentaron un mayor contenido, en promedio, que los requerimientos mínimos de cada especie.

La información disponible con relación a los requerimientos minerales de especies como el hámster, peces ornamentales y tortugas, son escasos, lo cual impidió realizar un análisis integral de la información desarrollada. Sin embargo, la información descrita y recopilada, se puede utilizar como punto de partida en el manejo de la nutrición mineral de animales de interés zootécnico.

\section{Agradecimientos}

Se agradece al Centro de Investigaciones en Nutrición Animal (CINA) de la Universidad de Costa Rica, por las facilidades y el apoyo brindado durante la ejecución de este proyecto. A la Vicerrectoría de Investigación de la Universidad de Costa Rica por el financiamiento brindado al proyecto VI-739-B8019 Cumplimiento del análisis de garantía en alimentos importados para mascotas.

\section{Referencias}

Association of Official Analytical Chemists International. (2005). Official methods of analysis of AOAC International (18 ${ }^{\text {th }}$ Ed.). Association of Official Analytical Chemists International.

Asamblea Legislativa de la República de Costa Rica. (1994, 13 diciembre). Ley 7451. Ley de bienestar de los animales. Procuraduría General de la República. http://www.pgrweb.go.cr/scij/Busqueda/Normativa/Normas/nrm_texto_ completo.aspx?nValor1=1\&nValor2=24319

Campos Granados, C. (2015). El impacto de los micronutrientes en la inmunidad de los animales. Nutrición Animal Tropical, 9(1), 1-23. https://doi.org/10.15517/nat.v9i1.18778

Case, L. P., Carey, D. P., \& Hirakawa, D. A. (1997). Canine and feline nutrition a resource for companion animal professionals. Hartcourt Brace.

Chen, C. -Y., Chen, S. -M., \& Huang, C. -H. (2014). Dietary magnesium requirement of soft-shelled turtles, Pelodiscus sinensis, fed diets containing exogenous phytate. Aquaculture, 432(1), 80-84.https://doi.org/10.1016/j.aquaculture.2014.05.001

Agron. Mesoam. 33(2): Artículo 47078, 2022

ISSN 2215-3608 doi:10.15517/am.v33i2.47078 
Corcoran, M., \& Roberts-Sweeney, H. (2014). Aquatic animal nutrition for the exotic animal practitioner. Veterinary Clinics of North America - Exotic Animal Practice, 17(3), 333-346. https://doi.org/10.1016/j.cvex.2014.05.005

Davies, M., Alborough, R., Jones, L., Davis, C., Williams, C., \& Gardner, D. S. (2017). Mineral analysis of complete dog and cat foods in the UK and compliance with European guidelines. Scientific Reports, 7(1), Article 17107. https://doi. org/10.1038/s41598-017-17159-7

de Blas, C., \& Wiseman, J. (2010). Nutrition of the rabbit (2 ${ }^{\text {nd }}$ Ed.). CABI. https://doi.org/10.1079/9781845936693.0000

Donoghue, S. (1998). Nutrition of pet amphibians and reptiles. Seminars in Avian and Exotic Pet Medicine, 7(3), $148-153$. https://doi.org/10.1016/s1055-937x(98)80006-6

Donoghue, S., \& McKeown, S. (1999). Nutrition of captive reptiles. The Veterinary Clinics of North America. Exotic Animal Practice, 2(1), 69-91. https://doi.org/10.1016/S1094-9194(17)30140-8

Fuller, M. (2004). Encyclopedia of farm animal nutrition. CABI. https://doi.org/10.1079/9780851993690.0000

Gagné, J. W., Wakshlag, J. J., Center, S. A., Rutzke, M. A., \& Glahn, R. P. (2013). Evaluation of calcium, phosphorus, and selected trace mineral status in commercially available dry foods formulated for dogs. Journal of the American Veterinary Medical Association, 243(5), 658-666. https://doi.org/10.2460/javma.243.5.658

Goff, J (2018). Mineral absorption mechanisms, mineral interactions that affect acid-base and antioxidant status, and diet considerations to improve mineral status. Journal Dairy Science, 101(1), 2763-2813. https://doi.org/10.3168/ jds.2017-13112

Gómez, L. F., Atehortua, C. G., \& Orozco, S. C. (2007). The influence of mascots in human lives. Revista Colombiana de Ciencias Pecuarias, 20(3), 377-386.

Hand, M., Thatcher, C., Remillard, R. L., \& Roudebush, P. (2000). Small animal clinical nutrition (4 ${ }^{\text {th }}$ Ed.). Mark Morris Institute.

Hendriks, W. H., Bakker, E. J., \& Bosch, G. (2015). Protein and amino acid bioavailability estimates for canine foods. Journal of Animal Science, 93(10), 4788-4795. https://doi.org/10.2527/jas.2015-9231

Lall, S. P., \& Lewis-McCrea, L. M. (2007). Role of nutrients in skeletal metabolism and pathology in fish - An overview. Aquaculture, 267(1-4), 3-19. https://doi.org/10.1016/j.aquaculture.2007.02.053

Lauten, S. D., Cox, N. R., Brawner, W. R., Goodman, S. A., Hathcock, J. T., Montgomery, Kincaid, S., Morrison, N., Spano, J., Lepine, A., Reinhart, G., \& Baker, H. J. (2002). Influence of dietary calcium and phosphorus content in a fixed ratio on growth and development in Great Danes. American Journal of Veterinary Research, 63(7), 1036-1047. https://doi. org/10.2460/ajvr.2002.63.1036

Lim, C., \& Klesius, P. H. (2019). El papel de los minerales traza en la salud de los peces. Avances en Nutrición Acuícola: Memorias del Cuarto Simposium Internacional de Nutrición Acuícola, 1998, 270-281. https://nutricionacuicola.uanl. $\mathrm{mx} /$ index.php/acu/article/view/303/0

Llínas-Solano, H. L., \& Rojas-Álvarez, C. R. (2005). Estadística descriptiva y distribuciones de probabilidad. Editorial Universidad del Norte. https://editorial .uninorte.edu.co/gpd-estadistica-descriptiva-y-distribuciones-de-probabilidad.html

Ministerio de Agricultura y Ganadería de Costa Rica. (2021, abril). Programa de registro y control de calidad de alimentos para animales. FEEDNET. http://feednet.senasa.go.cr/consulta/index.htm 
Ministerio de Comercio Exterior, Ministerio de Economía, Industria y Comercio, \& Ministerio de Agricultura y Ganadería. (2012). Reglamento Técnico Centroamericano RTCA 65.05.52:11. Productos utilizados en alimentación animal y establecimientos requisitos de registro sanitario y control. Procuraduría General de la República. http://www.pgrweb. go.cr/scij/Busqueda/Normativa/Normas/nrm_texto_completo.aspx?param1=NRTC\&nValor1=1\&nValor2=73269\&n Valor3=89862\&strTipM=TC

Mulder, G. B. (2012). Chapter 28 - Management, husbandry, and colony health. In M. A. Suckow, K. A Steves, \& R. P. Wilson (Eds.), The laboratory rabbit, guinea pig, hamster, and other rodents (1 ${ }^{\text {st }}$ Ed., pp. 765-777). Elsevier. https://doi. org/10.1016/B978-0-12-380920-9.00028-6

National Research Council. (1977). Nutrient Requirements of Rabbits (2 ${ }^{\text {nd }}$ Ed.). The National Academies Press. https://doi. $\operatorname{org} / 10.17226 / 35$

National Research Council. (1995). Nutrient Requirements of Laboratory Animals (4 ${ }^{\text {th }}$ Ed.). The National Academies Press. https://doi.org/10.17226/4758

National Research Council. (2006). Nutrient requirements of dogs and cats. The National Academies Press. https://doi. org/10.17226/10668

Rawski, M., Mans, C., Kierończyk, B., Świątkiewicz, S., Barc, A., \& Józefiak, D. (2018). Freshwater turtle nutrition - A review of scientific and practical knowledge. Annals of Animal Science, 18(1), 17-37. https://doi.org/10.1515/aoas-2017-0025

Remillard, R. L. (2008). Homemade diets: Attributes, pitfalls, and a call for action. Topics in Companion Animal Medicine, 23(3), 137-142. https://doi.org/10.1053/j.tcam.2008.04.006

Ricci, R., Sartori, A., Palagiano, C., \& Dalle Zotte, A. (2010). Study on the nutrient adequacy of feeds for pet rabbits available in the italian market. World Rabbit Science, 18(3), 131-137. https://doi.org/10.4995/wrs.2010.7532

Sales, J., \& Janssens, G. P. J. (2003). Nutrient requirements of ornamental fish. Aquatic Living Resources, 16(6), 533-540. https://doi.org/10.1016/j.aquliv.2003.06.001

Tal, M., Parr, J. M., MacKenzie, S., \& Verbrugghe, A. (2018). Dietary imbalances in a large breed puppy, leading to compression fractures, vitamin D deficiency, and suspected nutritional secondary hyperparathyroidism. Canadian Veterinary Journal, 59(1), 36-42. https://pubmed.ncbi.nlm.nih.gov/29302100/

Velasco-Santamaría, Y., \& Corredor-Santamaría, W. (2011). Nutritional requirements of freshwater ornamental fish: A review. Revista MVZ Córdoba, 16(2), 2458-2469. https://doi.org/10.21897/rmvz.283

Agron. Mesoam. 33(2): Artículo 47078, 2022

ISSN 2215-3608 doi:10.15517/am.v33i2.47078 